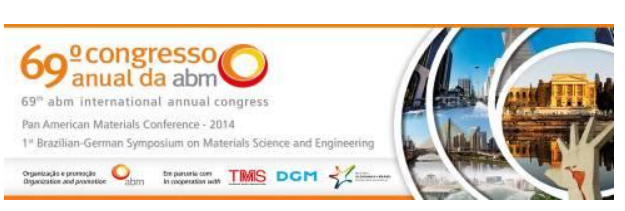

Tema: Produtos metálicos ferrosos

\title{
DESENVOLVIMENTO DE AÇO API 5L-X65 PARA DUTOS RESISTENTES AO SERVIÇO ÁCIDO (SOUR SERVICE)*
}

\section{Resumo}

\author{
Ricardo Miranda Alé1 \\ Carmos Antônio Gandra ${ }^{2}$ \\ Odair José dos Santos ${ }^{3}$ \\ Ronaldo Silva ${ }^{4}$ \\ Fabio Arroyo 5 \\ Marcelo Salanit \\ Carlos Jose Bandeira de Mello Joia \\ Ilson Palmieri Batista ${ }^{8}$ \\ Vinicius Rabello de Abreu Lima $^{9}$
}

Aços resistentes ao serviço ácido (sour service), com requisitos adicionais de alta resistência mecânica e tenacidade, têm sido especificados para aplicação em gasodutos do Pólo Pré-sal da Bacia de Santos. O desenvolvimento de aços que atendam a estes severos requisitos, visando à fabricação de tubos de elevada espessura, constitui um enorme desafio para o fabricante do aço. No presente trabalho, é apresentado o desenvolvimento do aço API 5L-X65 Sour Service para esse tipo de aplicação, onde são descritas as principais ações realizadas no processo de produção que permitiram o sucesso no desenvolvimento do produto, confirmado pelas excelentes propriedades obtidas nas chapas e nos tubos.

Palavras-chave: Aço grau X65; Serviço ácido; Gasoduto, Pré-sal.

\section{DEVELOPMENT OF API 5L X-65 PIPELINE STEEL FOR SOUR SERVICE APPLICATION}

\begin{abstract}
Steels for sour service application, with additional requirements for high strength and toughness, have been specified for use in the Santos Basin pre-salt gas pipelines. The development of steels that meet these stringent requirements, aiming at the fabrication of pipes with high thickness, is an enormous challenge for the steel manufacturers. This paper presents the development of API 5L-X65 Sour Service for this application, which describes the main actions carried out in the production process that enabled the successful development.
\end{abstract}

Keywords: X65 steel grade; Sour service; Linepipe, Pre-salt.

\footnotetext{
Eng. Metalurgista, M.Sc., D.Sc., Especialista de Produto, Usiminas, Belo Horizonte, MG, Brasil. Engenheiro Metalurgista, M.Sc., Engenheiro de Produto Sênior, Usiminas, Ipatinga, MG. Brasil. Engenheiro Metalurgista, M.Sc., D.Sc., Pesquisador Especialista, Usiminas, Ipatinga, MG, Brasil. Engenheiro Mecânico, M.Sc. - Gerente de Engenharia de Produto, Tenaris, Pindamonhangaba, $S P$, Brasil.

5 Eng. Mecânico, Coord. de Desenvolvimento de Produtos, Tenaris, Pindamonhangaba, SP, Brasil.

6 Engenheiro Metalurgista, M.Sc., Gerente de Metalurgia, Tenaris, Pindamonhangaba, SP, Brasil.

7 Engenheiro Metalurgista, Consultor Master, Petrobras, Rio de Janeiro, RJ, Brasil.

8 Engenheiro Metalurgista, MSc, Consultor, Petrobras, Rio de Janeiro, RJ, Brasil.

9 Engenheiro Mecânico, Engenheiro de Equipamentos, Petrobras, Rio de Janeiro, RJ, Brasil.
}

\footnotetext{
* Contribuição técnica ao 69ำ Congresso Anual da ABM - Internacional e ao 14ํㅡㄹ ENEMET - Encontro Nacional de Estudantes de Engenharia Metalúrgica, de Materiais e de Minas, 21 a 25 de julho de 2014, São Paulo, SP, Brasil.
} 


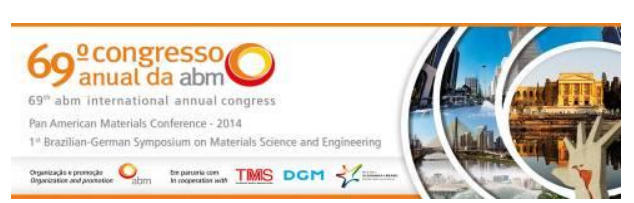

\section{INTRODUÇÃO}

As reservas de óleo e gás natural podem conter algum nível de $\mathrm{H}_{2} \mathrm{~S}$ (conhecido como gás sour) e este gás, na presença de umidade, pode ser crítico para os dutos metálicos. No caso do aço, o hidrogênio gerado no processo corrosivo pode determinar a ocorrência de dois tipos de trincas, conhecidas pelas abreviaturas SSC (Sulfide Stress Cracking) e HIC (Hydrogen Induced Cracking). Embora o mecanismo de SSC seja o principal problema relacionado à possibilidade de falha pelo $\mathrm{H}_{2} \mathrm{~S}$, $\mathrm{o}$ mecanismo de HIC também deve ser evitado, pois estas trincas podem, com o tempo, ocasionar a falha do duto. No ponto de vista do fabricante do aço, a obtenção de resistência ao HIC apresenta um nível de complexidade maior que a de resistência à SSC, sendo comum tratar os aços resistentes ao serviço ácido (sour service) como aços resistentes ao HIC.

Os aços resistentes ao HIC podem apresentar restrições com relação ao cumprimento, ao mesmo tempo, de outros requisitos, como valores elevados de resistência mecânica e de tenacidade. O cumprimento de todos estes requisitos é um enorme desafio a ser superado pelo fabricante do aço, especialmente em tubos de elevada relação espessura/diâmetro (t/D), onde as chapas são sujeitas a um alto nível de deformação a frio, durante a conformação dos tubos.

Neste trabalho são apresentados os resultados do desenvolvimento do aço API 5LX65 Sour Service, conduzido pela Usiminas em conjunto com a Petrobras e a TenarisConfab.

\subsection{Resistência ao HIC}

A nucleação de HIC ocorre, principalmente, em inclusões não metálicas, sendo as inclusões alongadas de MnS consideradas as mais nocivas [1]. Com isso, a susceptibilidade ao HIC diminui com a redução do teor de enxofre e com o controle da forma das inclusões, através do tratamento com cálcio. Normalmente, são indicados teores de enxofre abaixo de 10 ppm e uma relação de $C a / S \geq 2$ para se evitar o problema [1,2]. Além disso, para garantir uma elevada limpidez, sugere-se que o teor de oxigênio seja mantido abaixo de 25ppm [1].

Precipitados grosseiros, de TiN [3], de $(\mathrm{Ti}, \mathrm{Nb})(\mathrm{C}, \mathrm{N})[1,4]$ e de $\mathrm{Nb}(\mathrm{C}, \mathrm{N})[3,5]$, são também reportados na literatura como sítios potenciais para a nucleação de HIC. Os precipitados de TiN começam a se formar na fase líquida e coalescem rapidamente, em função das altas taxas de difusão nessa fase. Para se evitar a formação de precipitados grosseiros de TiN, a concentração de $\mathrm{Ti}$ deve ficar abaixo da relação estequiométrica, numa faixa considerada ótima $(2,0 \sim 3,5)$ [6]. No caso de precipitados grosseiros de $(\mathrm{Ti}, \mathrm{Nb})(\mathrm{C}, \mathrm{N})$, ocorre agregação do $\mathrm{Nb}$ à interface matriz/partícula de $\mathrm{Ti}(\mathrm{C}, \mathrm{N})$ [4], sendo a sua formação significativamente reduzida pela diminuição do teor de Ti para valores da ordem de 0,012\% [7]. Já a formação de precipitados grosseiros de $\mathrm{Nb}(\mathrm{C}, \mathrm{N})$ é favorecida pela elevada tendência do nióbio em segregar no centro da espessura das placas de lingotamento contínuo [3]. Em função disso, alguns autores [5] limitam o teor máximo de $\mathrm{Nb}$ em 0,035\%.

Normalmente, o HIC se propaga em fases de dureza mais elevada no centro da espessura de chapas produzidas via lingotamento contínuo, na região de segregação central. Nesse aspecto, a utilização de um baixo teor de carbono é essencial, já que o nível de segregação durante a solidificação é menor com o estreitamento do intervalo entre as temperaturas liquidus e solidus e ampliação do campo de temperatura da região de ferrita $\delta$ do diagrama Fe-C [2]. Outros elementos

\footnotetext{
*Contribuição técnica ao $69^{\circ}$ Congresso Anual da ABM - Internacional e ao 14ํㅡㄹ ENEMET - Encontro Nacional de Estudantes de Engenharia Metalúrgica, de Materiais e de Minas, 21 a 25 de julho de 2014, São Paulo, SP, Brasil.
} 


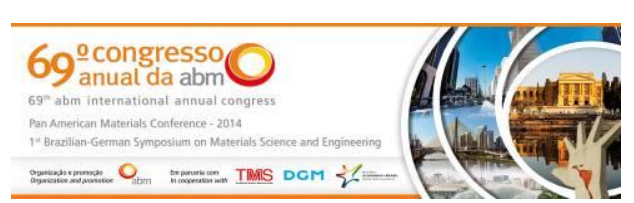

relevantes no contexto da segregação central são o fósforo, devido ao seu coeficiente de segregação mais elevado, e o manganês, que apresenta um maior teor comparativamente aos demais elementos de liga presentes em aços de alta resistência microligados para dutos [8]. Consequentemente, o fósforo é mantido em teores baixos, tipicamente menores que 0,012\% [8]. Já o teor de manganês tolerável para evitar o HIC é tão maior quanto menor o teor de carbono do aço, mas deve ser limitado em 1,7\%, mesmo para um teor de carbono da ordem de 0,03\% [9].

A redução do teor de carbono é, entretanto, limitada, pois pode comprometer a obtenção de resistência mecânica em aços produzidos via resfriamento acelerado de maior espessura, tendo em vista a redução da temperabilidade. Assim, a segregação central em placas deve também ser reduzida através da otimização de parâmetros do processo de lingotamento contínuo, tais como: nível de superaquecimento, velocidade de lingotamento, taxa de resfriamento secundário, ajuste dos rolos, etc [2].

Além disso, o emprego do resfriamento acelerado, após a etapa de laminação controlada, é essencial para a fabricação de aços resistentes ao HIC, pois contribui para que o carbono fique distribuído de forma mais uniforme ao longo da espessura e a microestrutura se torne bastante homogênea. Isso ocorre com a redução da difusão do carbono para a austenita ainda não transformada, que se concentra na região de segregação central, devido à maior taxa de resfriamento da chapa. Com relação à microestrutura, há uma tendência geral na literatura de indicar que a ferrita acicular fornece a melhor resistência ao HIC [2,10]. De acordo com Park e outros [11], esse comportamento pode ser explicado pela elevada tenacidade desse constituinte, que impede a propagação da trinca.

\subsection{Propriedades Mecânicas}

Uma microestrutura constituída predominentemente de ferrita acicular garante ao aço, além de boa resistência ao HIC, a melhor combinação desejada de alta resistência mecânica e excelente tenacidade ${ }^{(12)}$, sendo portanto visada para aços do grau X65 para serviço ácido. Essa característica de boas propriedades mecânicas da ferrita acicular é atribuída à presença de uma densidade de discordâncias relativamente elevada e granulação fina(13). Essa microestrutura só pode ser obtida com a utilização do resfriamento acelerado, sendo favorecida para maiores taxas de resfriamento e menores temperaturas de final de resfriamento ${ }^{(14)}$.

Considerando que os aços resistentes ao HIC apresentam baixo teor de carbono e limitado teor de manganês, a fim de alcançar a microestrutura e propriedades mecânicas desejadas são realizadas, normalmente, adições de elementos de liga, tais como: $\mathrm{Nb}, \mathrm{V}, \mathrm{Ti}, \mathrm{Mo}, \mathrm{Ni}$, Cu e $\mathrm{Cr}^{(14)}$.

\section{PROCEDIMENTO EXPERIMENTAL}

O desenvolvimento do aço API 5L-X65 Sour Service foi realizado através de experiências em escala industrial, para atendimento à especificação da Petrobras referente à fabricação de tubos de 24" de diâmetro, em espessuras de até $32,45 \mathrm{~mm}$, visando à aplicação em gasodutos do Pólo Pré-sal da Bacia de Santos, dos projetos Rotas 2 e 3 da Petrobras. Foram produzidas diversas corridas, em quatro diferentes ciclos, mostrados esquematicamente na figura 1.

\footnotetext{
*Contribuição técnica ao $69^{\circ}$ Congresso Anual da ABM - Internacional e ao 14ํㅡㄹ ENEMET - Encontro Nacional de Estudantes de Engenharia Metalúrgica, de Materiais e de Minas, 21 a 25 de julho de 2014, São Paulo, SP, Brasil.
} 


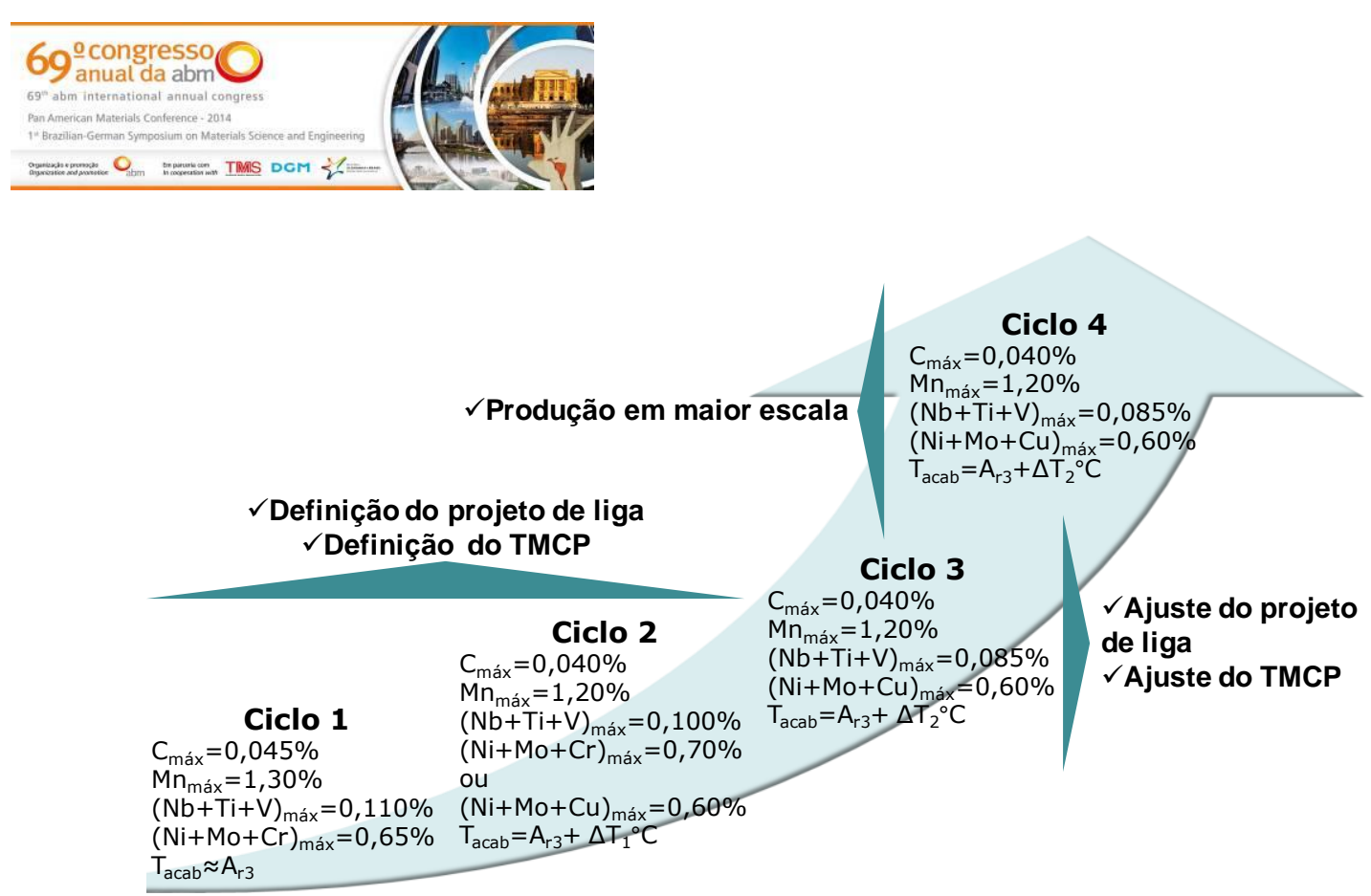

Figura 1. Representação esquemática da evolução do desenvolvimento do aço API X65 Sour Service

Nos ciclos 1 e 2 foi buscada a definição do projeto de liga e das condições de processamento termomecânico (TMCP). No ciclo 3 obteve-se a concepção final do produto, com ajustes finos no projeto de liga e nas condições de processamento termomecânico. Para confirmação da qualidade do produto obtido, foram enviadas para a TenarisConfab cerca de $100 \mathrm{t}$ de chapas do ciclo 3 para transformação em tubos. No ciclo 4 foi verificada a robustez do projeto com a produção em maior escala desse aço, com base na concepção obtida no ciclo 3. Nesse ciclo foram realizados ajustes na rota de aciaria, no intuito de garantir um melhor acerto de composição química, evitando uma dispersão nas propriedades do aço.

O desenvolvimento de aços em escala industrial na Usiminas é feito utilizando uma ampla base de dados operacionais e resultados de estudos de pesquisa. Complementarmente são utilizadas ferramentas de simulação numérica para suporte a este desenvolvimento, particularmente na definição e ajuste do projeto de liga, como apresentado nos próximos itens.

\subsection{Projeto de Liga}

O princípio adotado, na definição do projeto de liga, foi o de se estabelecer um balanço entre a redução na possibilidade de formação de fases de elevada dureza e de precipitados grosseiros, na região de segregação central, e a obtenção do nível de resistência mecânica especificado. A tabela 1 apresenta as restrições preliminares de composição química utilizadas no ciclo 1. Em função da influência prejudicial na resistência ao HIC e nas propriedades mecânicas, foram utilizados os teores mais baixos possíveis de enxofre, fósforo e oxigênio.

Tabela 1. Composição química especificada para o ciclo 1

\begin{tabular}{c|c|c|c|c|c|c|c|c}
\hline $\mathrm{C}(\%)$ & $\mathrm{Mn}(\%)$ & $\mathrm{P}(\mathrm{ppm})$ & $\mathrm{S}(\mathrm{ppm})$ & $\mathrm{O}(\mathrm{ppm})$ & $\begin{array}{c}\mathrm{Ti}+\mathrm{Nb}+\mathrm{V} \\
(\%)\end{array}$ & $\begin{array}{c}\mathrm{Cr}+\mathrm{Cu}+\mathrm{Ni}+\mathrm{Mo} \\
(\%)\end{array}$ & $\mathrm{Ca} / \mathrm{S}$ & $\mathrm{Ti} / \mathrm{N}$ \\
\hline$\leq 0,045$ & $\leq 1,30$ & $\leq 150$ & $\leq 12$ & $\leq 30$ & $\leq 0,110$ & $\leq 0,70$ & $\geq 2$ & 2,0 a 3,5 \\
\hline
\end{tabular}

A redução dos teores de carbono e manganês, a partir do ciclo 2, visou a minimizar o nível de segregação central e teve como base a influência dessa redução no estreitamento do intervalo entre as temperaturas liquidus e solidus e ampliação do campo de temperatura da região de ferrita $\delta$, verificado no diagrama Fe-C (figura 2).

\footnotetext{
* Contribuição técnica ao 69ำ Congresso Anual da ABM - Internacional e ao 14ํㅡㄹ ENEMET - Encontro Nacional de Estudantes de Engenharia Metalúrgica, de Materiais e de Minas, 21 a 25 de julho de 2014, São Paulo, SP, Brasil.
} 

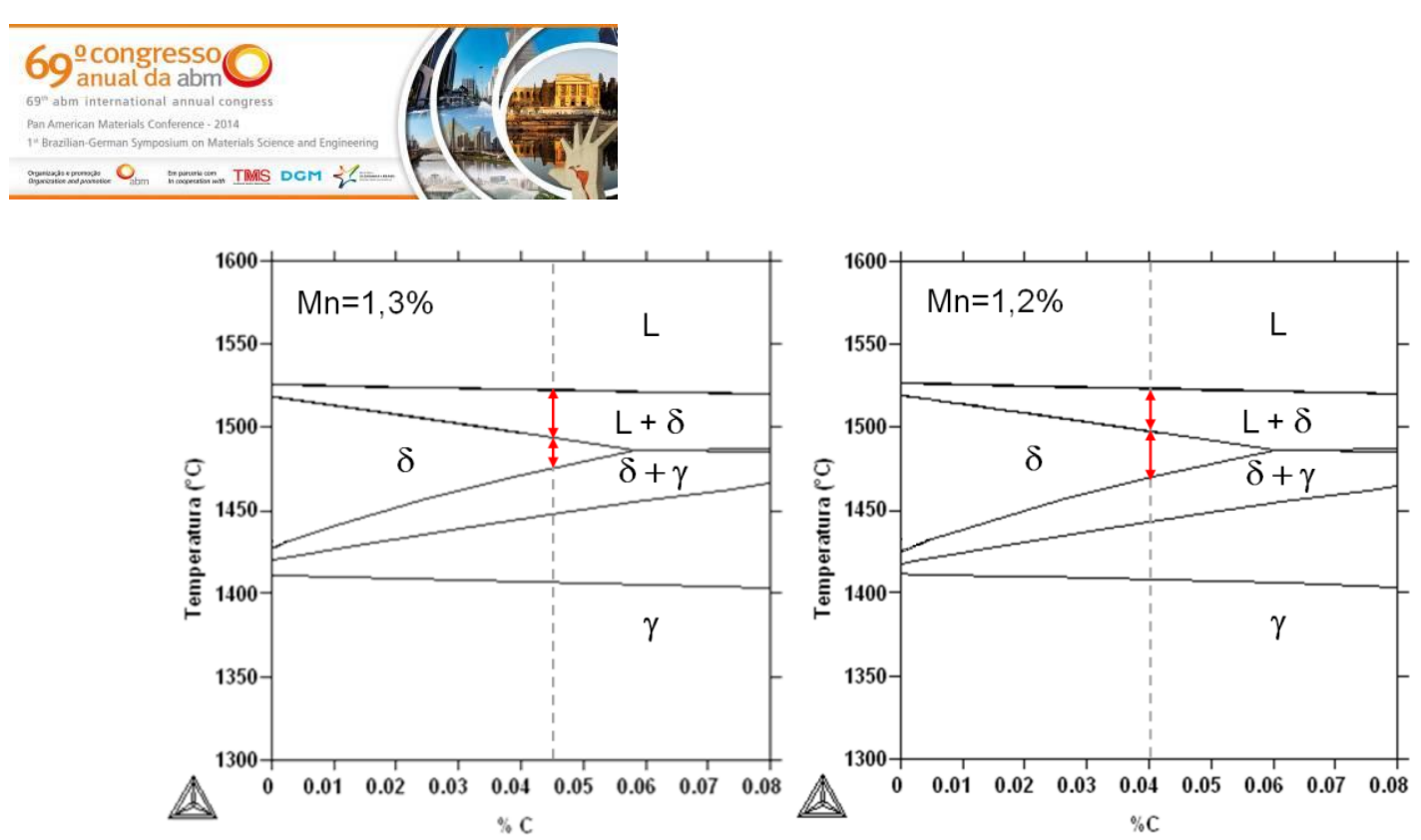

Figura 2. Diagrama Fe-C simulado numericamente utilizando o Thermo-Calc® de aços com diferentes teores de manganês

A figura 3 mostra a presença, em ensaio de HIC, de trinca nucleada em partícula grosseira de $(\mathrm{Ti}, \mathrm{Nb})(\mathrm{C}, \mathrm{N})$, identificada por mapeamento de raios- $\mathrm{X}$, observada em chapa oriunda de corrida do ciclo 2, que apresentou teor de Ti no limite superior do especificado. Com o objetivo de eliminar esse problema, foram reduzidos a partir do ciclo 3 os teores de elementos microligantes, particularmente de titânio e nióbio.
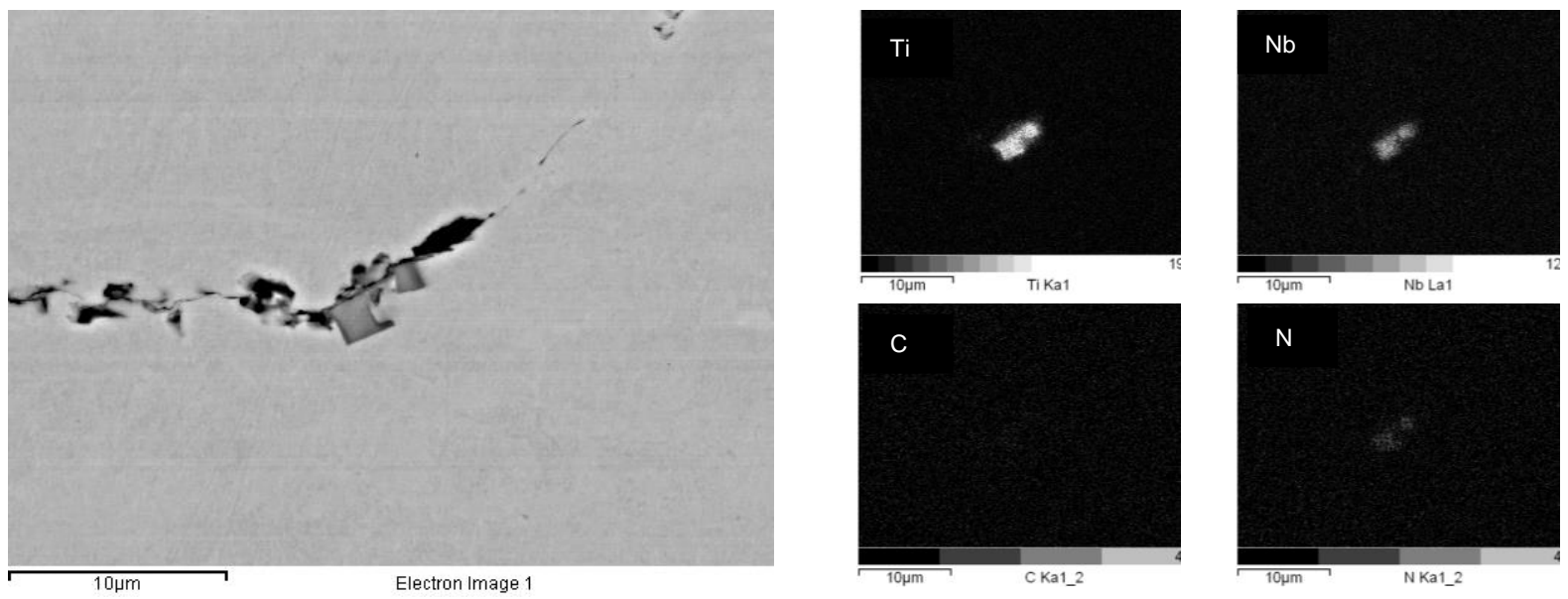

Figura 3. Trinca nucleada em partícula de $(\mathrm{Ti}, \mathrm{Nb})(\mathrm{C}, \mathrm{N})$ identificada por mapeamento de raios- $\mathrm{X}$

Como as partículas de $(\mathrm{Ti}, \mathrm{Nb})(\mathrm{C}, \mathrm{N})$ se formam em temperaturas muito elevadas, com a presença de fase líquida, estas tenderão a ser tanto mais grosseiras quanto menor a quantidade de fases sólidas no momento da precipitação. A figura 4 mostra a fração de fases sólidas no momento da precipitação do $(\mathrm{Ti}, \mathrm{Nb})(\mathrm{C}, \mathrm{N})$, indicada pelas setas, calculada utilizando o modelo de Scheil-Gulliver [7], para teores de $\mathrm{Ti}+\mathrm{Nb}=0,060 \%$ (a) e $\mathrm{Ti}+\mathrm{Nb}=0,033 \%$ (b), com os demais elementos constantes na faixa definida para o ciclo 3 . Fica evidente que a redução dos teores de $\mathrm{Ti}+\mathrm{Nb}$ de $0,060 \%$ para $0,033 \%$ determina a formação de partículas de $(\mathrm{Ti}, \mathrm{Nb})(\mathrm{C}, \mathrm{N})$ a uma temperatura onde a fração de fases sólidas é mais elevada, em mais de $5 \%$. Considerando que o modelo de Scheil-Gulliver tende a superestimar o intervalo de solidificação [7], a fração real de fases sólidas é ainda maior do que a prevista. Portanto, a tendência à formação de partículas de $(\mathrm{Ti}, \mathrm{Nb})(\mathrm{C}, \mathrm{N})$ pode ser reduzida com a redução dos teores de $\mathrm{Ti}$ e $\mathrm{Nb}$.

A opção da utilização de cobre em substituição ao cromo foi definida no ciclo 2, quando se verificou que a concepção com cobre foi menos sensível às variações

*Contribuição técnica ao $69^{\circ}$ Congresso Anual da ABM - Internacional e ao 14ํㅡㄹ ENEMET - Encontro Nacional de Estudantes de Engenharia Metalúrgica, de Materiais e de Minas, 21 a 25 de julho de 2014, São Paulo, SP, Brasil. 


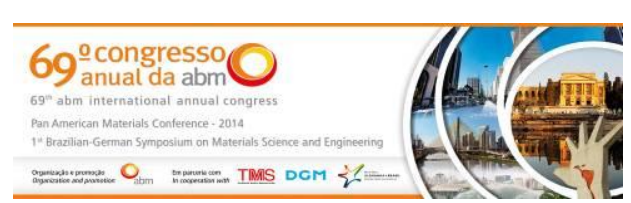

das condições de processamento termomecânico, para a obtenção dos níveis visados de propriedades mecânicas.
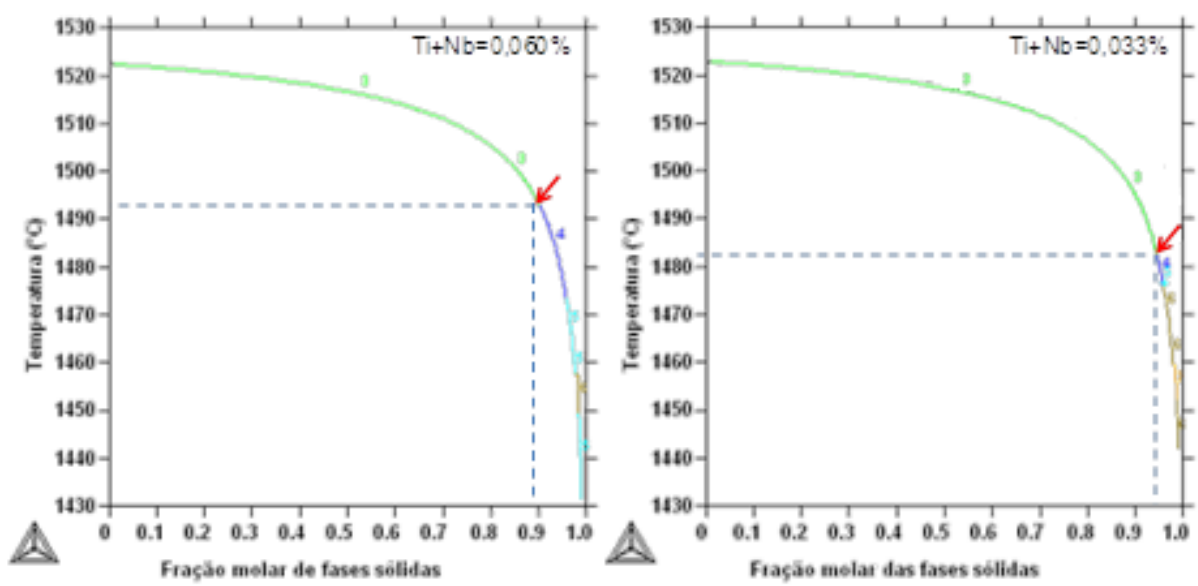

Figura 4. Fração molar de sólido como função da temperatura usando o modelo de Scheil- Gulliver simulado numericamente utilizando o Thermo-Calc®.

\subsection{Condições de Processo na Usiminas}

A figura 5 apresenta a rota utilizada na Aciaria da Usiminas para a obtenção de placas de aço de elevada limpidez, baixo teor de elementos residuais, faixa estreita de elementos de liga e baixo nível de segregação central. A figura mostra os objetivos metalúrgicos visados em cada etapa.

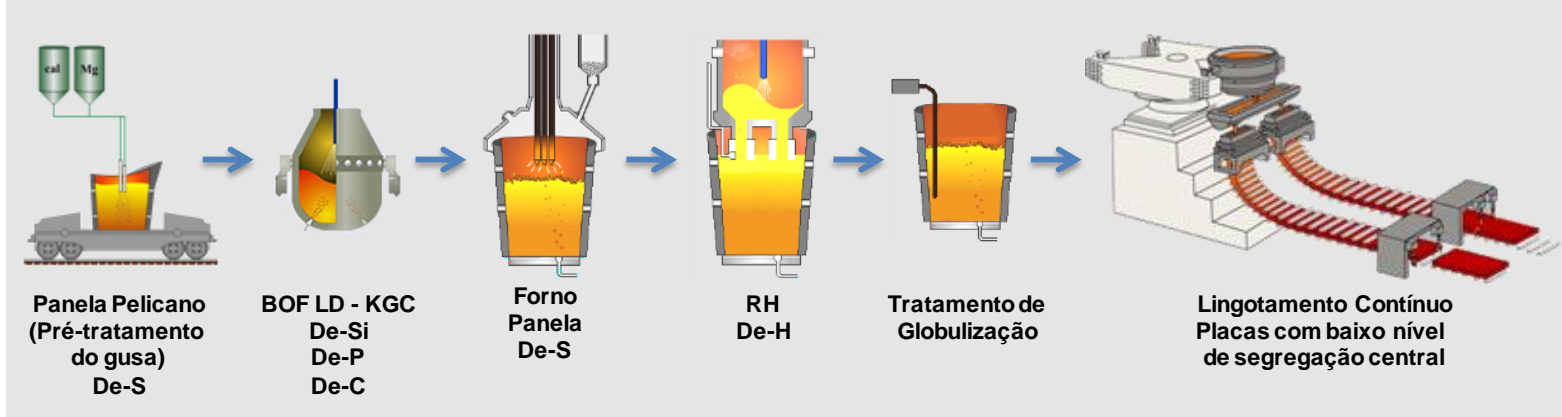

Figura 5. Representação esquemática da sequência otimizada na Aciaria da Usiminas para a produção de aço resistente ao HIC.

As condições de processamento termomecânicas foram definidas para a obtenção das propriedades mecânica em combinação com a resistência ao HIC desejada, utilizando diferentes mecanismos metalúrgicos. Os mais importantes mecanismos envolvidos neste caso são: (i) refino de grão - baixa temperatura de reaquecimento, laminação controlada com elevada redução na região de não recristalização da austenita e baixa temperatura final de acabamento e resfriamento acelerado após laminação controlada; (ii) endurecimento por precipitação - temperatura de reaquecimento suficientemente alta para solubilização dos precipitados de nióbio; e (iii) tipo e distribuição dos constituintes microestruturais - alta taxa de resfriamento acelerado, para obtenção de microestrutura homogênea com elevada proporção de ferrita acicular, e temperatura de fim de resfriamento suficientemente alta para evitar a formação de microconstituinte AM, mas suficientemente baixa para evitar uma baixa resistência mecânica.

* Contribuição técnica ao 69 Congresso Anual da ABM - Internacional e ao 14ํㅡㄹ ENEMET - Encontro Nacional de Estudantes de Engenharia Metalúrgica, de Materiais e de Minas, 21 a 25 de julho de 2014, São Paulo, SP, Brasil. 


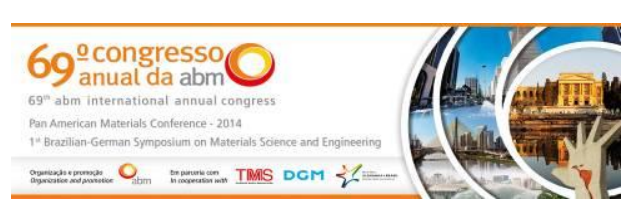

Durante o reaquecimento das placas, é desejável a dissolução da maior quantidade possível de nióbio na austenita, de modo que no resfriamento sejam obtidos carbonitretos finos, ricos em nióbio, dispersos na matriz. Esses precipitados são responsáveis por inibir a recristalização da austenita, tornando possível a obtenção de grãos muito finos, que conferem alta resistência mecânica e elevada tenacidade ao aço. Conforme pode ser visto na figura 6 , obtida utilizando o Thermo-Calc $\AA$ para a composição definida no ciclo 3, a temperatura de dissolução deste precipitado é da ordem de $1040^{\circ} \mathrm{C}$. Em função disso, adotou-se uma temperatura de reaquecimento superior a esse valor para o aço API 5L-X65 Sour Service.

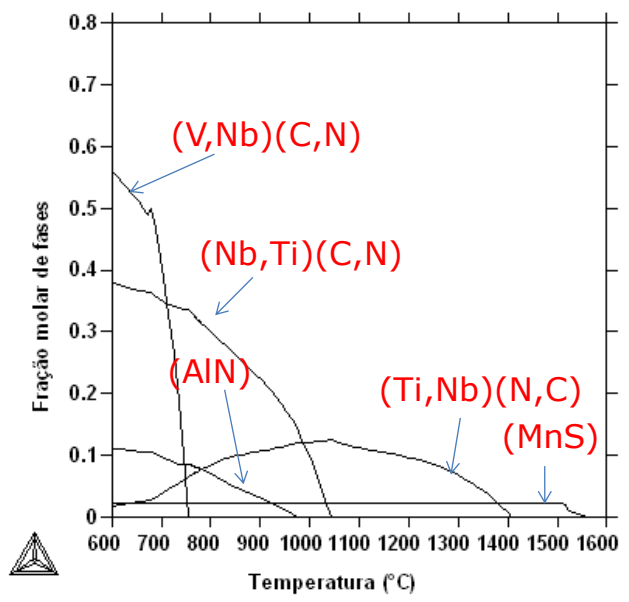

Figura 6. Diagrama com a fração molar de fases como função da temperatura, simulado numericamente utilizando o Thermo-Calc® de aço com composição química do ciclo 3

Na figura 7 é apresentado um diagrama CCT, construído para suporte à definição da temperatura final de acabamento da laminação controlada e da taxa de resfriamento acelerado. Os ciclos termomecânicos foram aplicados em material produzido em escala piloto com composição química dentro da faixa especificada na tabela 1 . A temperatura $\mathrm{Ar}_{3}$, obtida através da análise do diagrama, considerando a taxa de resfriamento de $0,5^{\circ} \mathrm{C} / \mathrm{s}$, é de aproximadamente $820^{\circ} \mathrm{C}$. Para o aço desenvolvido, a temperatura de final de laminação controlada adotada foi sempre superior a esse valor, de modo a obter os benefícios do resfriamento acelerado, com a microestrutura completamente austenítica no início do resfriamento. Com relação à taxa de resfriamento, foi definida a utilização de taxas entre $10^{\circ} \mathrm{C} / \mathrm{s}$ e $30^{\circ} \mathrm{C} / \mathrm{s}$, de modo a garantir a obtenção de uma microestrutura homogênea, com a maior quantidade de ferrita acicular possível.

\subsection{Fabricação dos Tubos}

Os tubos avaliados neste trabalho foram fabricados na TenarisConfab pelo processo UOE. Considerando a elevada deformação a frio na fabricação dos tubos relacionados a esse desenvolvimento ( $t / D \approx 5 \%$ ), a TenarisConfab buscou, através do controle dos parâmetros de processo, minimizar o nível de tensões residuais presente nos tubos, de modo a garantir a manutenção da tenacidade e da resistência ao HIC obtida nas chapas.

\subsection{Ensaios em Chapas e Tubos}

As chapas e os tubos foram avaliados em termos de composição química, propriedades mecânicas e resistência ao serviço ácido. A análise química e as

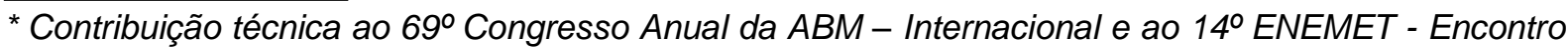
Nacional de Estudantes de Engenharia Metalúrgica, de Materiais e de Minas, 21 a 25 de julho de 2014, São Paulo, SP, Brasil. 


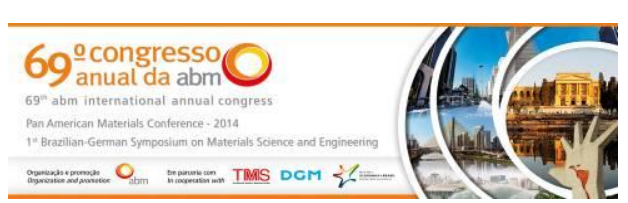

propriedades mecânicas (ensaio de tração, Charpy e DWTT) foram avaliadas de acordo com a norma API 5L [14]. A resistência ao serviço ácido foi avaliada através do ensaio de HIC, de acordo com a norma DNV-OS-F101 [15]. Complementarmente, uma chapa e um tubo foram submetidos aos ensaios de CTOD e de corrosão sob tensão (SSC - Sulfide Stress Corrosion). Esses ensaios foram realizados de acordo com as normas BS-7448 [16] e NACE TM 0177 [17], respectivamente.

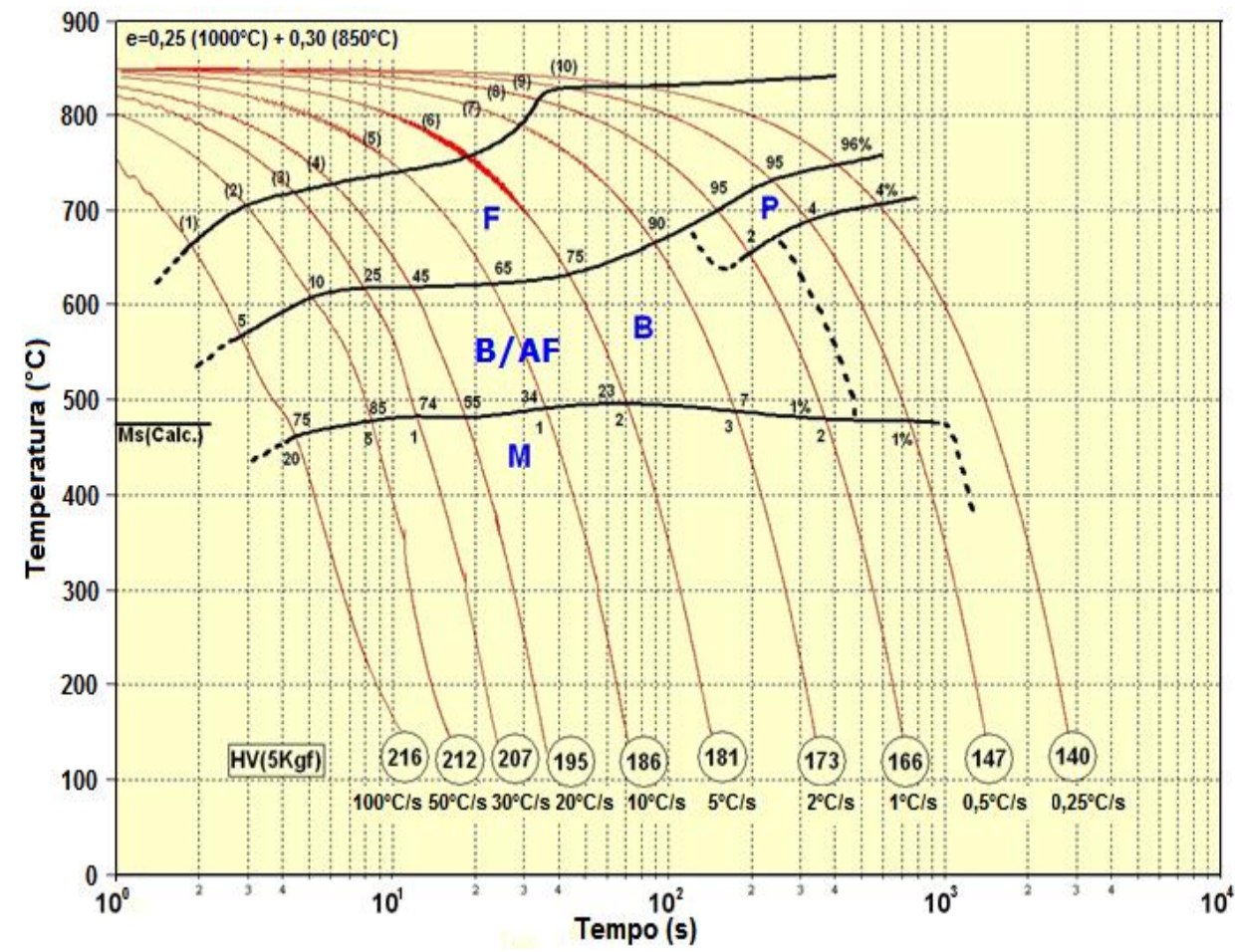

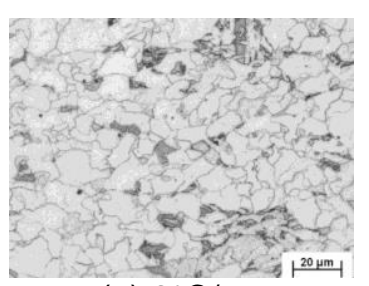

(a) $2^{\circ} \mathrm{C} / \mathrm{s}$

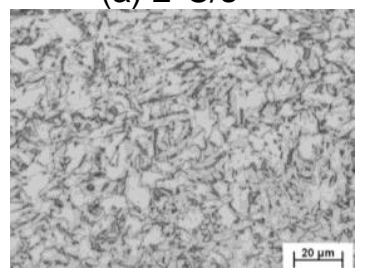

(b) $10^{\circ} \mathrm{C} / \mathrm{s}$

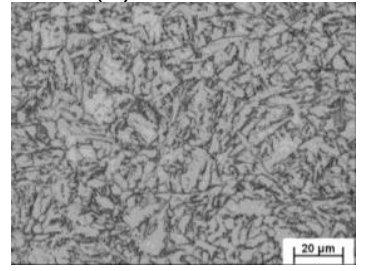

(c) $30^{\circ} \mathrm{C} / \mathrm{s}$

Figura 7. Curva CCT e microestrutura obtida para diferentes taxas de resfriamento

\section{RESULTADOS E DISCUSSÃO}

A composição química média das chapas produzidas no ciclo 3 e transformadas em tubos de 24" de diâmetro, nas espessuras de 29,65 mm e $32,45 \mathrm{~mm}$, são apresentadas na tabela 2. Destaca-se os baixos teores de carbono e carbono equivalente, além dos baixos teores de impurezas como o fósforo e o enxofre.

Tabela 2. Composição química média do aço API 5L-X65 Sour Service - ciclo 3

\begin{tabular}{c|c|c|c|c|c|c|c}
\hline $\mathrm{C}(\%)$ & $\mathrm{Mn}(\%)$ & $\mathrm{P}(\%)$ & $\mathrm{S}(\%)$ & $\begin{array}{c}\mathrm{Nb}+\mathrm{V}+\mathrm{T} \\
\mathrm{i} \\
(\%)\end{array}$ & $\begin{array}{c}\mathrm{Cu}+\mathrm{Ni}+\mathrm{Mo} \\
(\%)\end{array}$ & $\mathrm{Ceq}^{(1)}$ & $\mathrm{Pcm}^{(2)}$ \\
\hline 0,035 & 1,20 & 0,013 & 0,0010 & 0,063 & 0,52 & 0,29 & 0,13 \\
\hline (1) Ceq $=\% \mathrm{C}+\% \mathrm{Mn} / 6+(\% \mathrm{Cr}+\% \mathrm{Mo}+\% \mathrm{~V}) / 5+(\% \mathrm{Ni}+\% \mathrm{Cu}) / 15$ \\
(2) $\mathrm{Pcm}=\% \mathrm{C}+\% \mathrm{Si} / 30+(\% \mathrm{Mn}+\% \mathrm{Cu}+\% \mathrm{Cr}) / 20+\% \mathrm{Ni} / 60+\% \mathrm{Mo} / 15+\% \mathrm{~V} / 10+5 . \% B$
\end{tabular}

Os requisitos e resultados de propriedades mecânicas obtidos nas chapas e nos tubos são mostrados na tabela 3. Os resultados dos ensaios de tração, Charpy e CTOD atenderam plenamente às faixas especificadas para chapas e tubos. Embora os valores de limites de escoamento e de resistência das chapas tenham sido relativamente baixos, a aprovação dos tubos foi garantida com o ganho obtido nessas propriedades com a deformação a frio a qual as chapas foram submetidas durante a fabricação desses tubos.

\footnotetext{
* Contribuição técnica ao 69ำ Congresso Anual da ABM - Internacional e ao 14ํㅡㄹ ENEMET - Encontro Nacional de Estudantes de Engenharia Metalúrgica, de Materiais e de Minas, 21 a 25 de julho de 2014, São Paulo, SP, Brasil.
} 


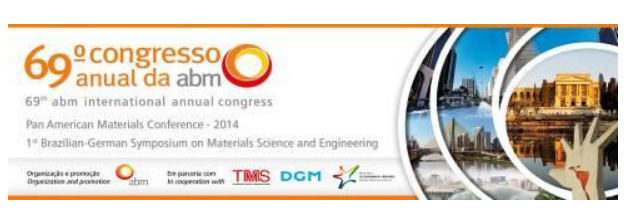

Tabela 3. Requisitos e resultados de propriedades mecânicas das chapas produzidas no ciclo 3

\begin{tabular}{|c|c|c|c|c|c|}
\hline \multirow{3}{*}{$\begin{array}{c}\begin{array}{c}\text { Propriedade } \\
\text { Mecânica }^{(1)}\end{array} \\
\text { Limite de Escoamento } \\
(\mathrm{MPa})\end{array}$} & \multirow{3}{*}{$\begin{array}{c}\text { Especificada } \\
\text { Tubo: } 450 \text { a } 570\end{array}$} & \multicolumn{4}{|c|}{ Obtida } \\
\hline & & \multicolumn{2}{|c|}{ Chapa } & \multicolumn{2}{|c|}{ Tubo } \\
\hline & & $\bar{X}=455$ & $\sigma=13$ & $\bar{X}=498$ & $\sigma=13$ \\
\hline $\begin{array}{l}\text { Limite de Resistência } \\
(\mathrm{MPa})\end{array}$ & Tubo: 540 a 760 & $\bar{X}=536$ & $\sigma=3$ & $\bar{X}=564$ & $\sigma=9$ \\
\hline Razão Elástica & $\begin{array}{c}\text { Chapa: Máximo 0,90 } \\
\text { Tubo: Máximo 0,92 }\end{array}$ & $\bar{X}=0,85$ & $\sigma=0,02$ & $\bar{X}=0,88$ & $\sigma=0,02$ \\
\hline $\begin{array}{c}\text { Alongamento (\%) } \\
\text { Base de Medida=2" }\end{array}$ & $\begin{array}{l}\text { Chapa: Mínimo } 30 \\
\text { Tubo: Mínimo } 25\end{array}$ & $\bar{X}=64,6$ & $\sigma=1,6$ & $\bar{X}=49,4$ & $\sigma=1,1$ \\
\hline Charpy (J) & $\begin{array}{l}\text { Chapa: } 150 \text { a }-40^{\circ} \mathrm{C} \\
\text { Tubo: } 150 \mathrm{a}-20^{\circ} \mathrm{C}\end{array}$ & $\bar{X}=454$ & $\sigma=4$ & $\bar{X}=432$ & $\sigma=13$ \\
\hline DWTT (\%Área Dúctil) & $\begin{array}{l}\text { Chapa: } 85 \mathrm{a}-20^{\circ} \mathrm{C} \\
\text { Tubo: } 85 \mathrm{a}-10^{\circ} \mathrm{C}\end{array}$ & $\bar{X}=97^{(2)}$ & $\sigma=5$ & $\bar{X}=98^{(2)}$ & $\sigma=4$ \\
\hline CTOD (mm) & $\begin{array}{l}\text { Chapa: } 0,40 \text { a }-20^{\circ} \mathrm{C} \\
\text { Tubo: } 0,15 \mathrm{a}-10^{\circ} \mathrm{C}\end{array}$ & \multicolumn{2}{|c|}{ 1,84 Mínimo } & \multicolumn{2}{|c|}{ 1,21 Mínimo } \\
\hline
\end{tabular}

Os resultados obtidos no ensaio DWT indicam que as chapas e tubos foram aprovados se desconsiderada a área de frágil da fratura inversa, observada em diversos corpos-de-prova. A fratura inversa é um tipo de fratura anormal no ensaio de DWT, que ocorre por clivagem na região de impacto do cutelo, oposta ao entalhe, mesmo com a fratura se propagando até essa região de forma dúctil. De acordo com a literatura [18,19], a fratura inversa pode ocorrer no ensaio de DWT em aços de elevada tenacidade. Para estes aços é consumida uma maior energia para a iniciação da fratura, determinando uma maior deformação plástica, e, consequentemente, um maior encruamento na região de impacto do cutelo, reduzindo a ductilidade desta região. Ainda não existe consenso sobre como tratar o problema da fratura inversa. Pesquisadores de siderúrgicas coreanas [18] e japonesas [19] sugerem desconsiderar a área de fratura inversa, como foi feito nesse trabalho na tabela 3 , tendo em vista que a região encruada não existe no aço na condição real de aplicação em dutos. Contudo, a recomendação API RP 5L3 [20] indica que a presença de fratura inversa torna o resultado do ensaio inválido. Assim, nesse trabalho, os resultados de DWTT que apresentaram fratura inversa foram desconsiderados e a tenacidade foi avaliada com base nos ensaios de CTOD e, principalmente, de Charpy. Os excelentes valores de tenacidade obtidos nesses ensaios foram associados à elevada limpidez do material e à presença de uma microestrutura muito fina, constituída principalmente de ferrita acicular, conforme mostrado na figura 8. A figura evidencia, também, que, além da microestrutura adequada, as condições de resfriamento acelerado foram bastante apropriadas para a obtenção de uma homogeneidade microestrutural ao longo da espessura.

Os requisitos e resultados de resistência à corrosão em serviço ácido são apresentados na tabela 4. Os ensaios de SSC foram realizados em apenas uma chapa e no tubo correspondente. Verifica-se que o aço API 5L-X65 Sour Service desenvolvido apresenta resistência ao HIC e à SSC, sendo, portanto, adequado para aplicação nas condições severas de serviço ácido. Esse comportamento foi associado ao baixo nível de segregação central e de inclusões de MnS apresentado pelo aço desenvolvido.

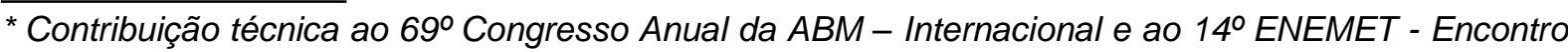
Nacional de Estudantes de Engenharia Metalúrgica, de Materiais e de Minas, 21 a 25 de julho de 2014, São Paulo, SP, Brasil.
} 


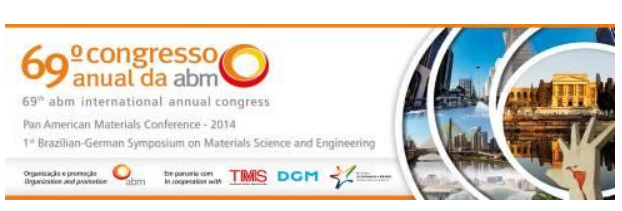

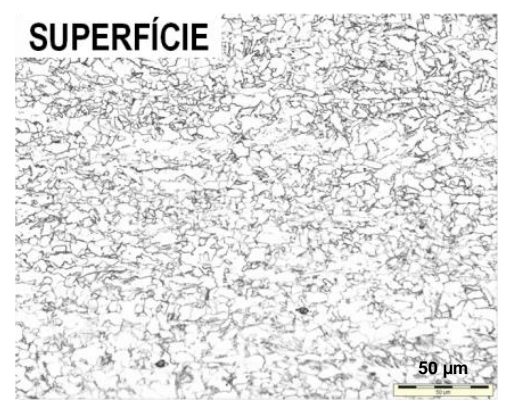

(a)

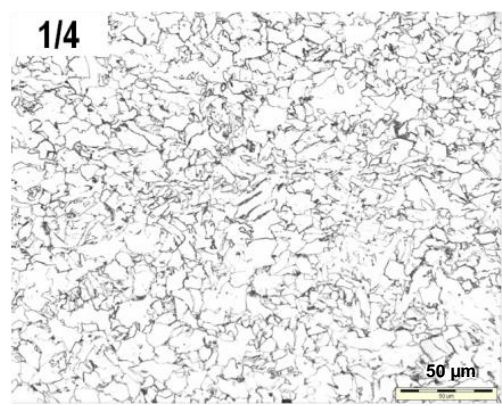

(b)

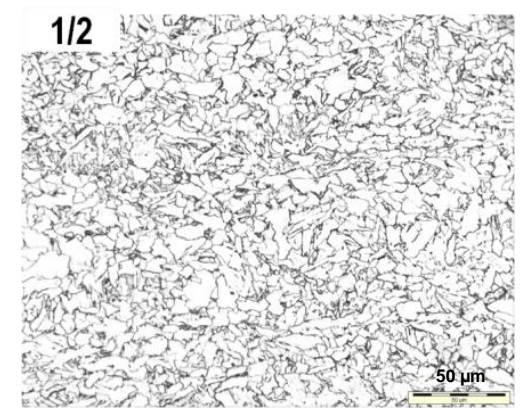

(c)

Figura 8. Microestrutura ao longo da espessura de chapa produzida no ciclo 3.

Tabela 4. Resultados de resistência à corrosão em serviço ácido de chapas produzidas no ciclo 3.

\begin{tabular}{|c|c|c|c|}
\hline \multirow{2}{*}{$\begin{array}{l}\text { Ensaio de Resistência } \\
\text { ao Serviço Ácido }\end{array}$} & \multirow{2}{*}{ Especificada } & \multicolumn{2}{|c|}{ Obtida } \\
\hline & & Chapa & Tubo \\
\hline $\begin{array}{c}\text { HIC } \\
\text { Solução B NACE TM0177 } \\
\left(\mathrm{pH}=3,5 \text { e } \mathrm{pH}_{2} \mathrm{~S}=1 \text { bar }\right)\end{array}$ & $\begin{array}{l}\text { CLR } \leq 15 \% \\
\text { CTR } \leq 5 \% \\
\text { CSR } \leq 2 \% \\
\end{array}$ & $\begin{array}{l}\text { Máximo } \mathrm{CLR}^{(*)}=7 \% \\
\text { Máximo CTR=0\% } \\
\text { Máximo CSR=0\% }\end{array}$ & $\begin{array}{l}\text { Máximo } \mathrm{CLR}^{\left({ }^{*}\right)}=3 \% \\
\text { Máximo } \mathrm{CTR}=0 \% \\
\text { Máximo CSR=0\% }\end{array}$ \\
\hline $\begin{array}{c}\text { SSC } \\
\text { Solução B NACE TM0177 } \\
\left.\text { (pH=3,5 e p } \mathrm{pH}_{2} \mathrm{~S}=1 \text { bar }\right)\end{array}$ & $\begin{array}{l}\text { Sem fratura e isento de } \\
\text { trincas (observação com } \\
\text { 10X de ampliação) }\end{array}$ & $\begin{array}{c}\text { Sem fratura e isento } \\
\text { de trincas }\end{array}$ & $\begin{array}{c}\text { Sem fratura e isento } \\
\text { de trincas }\end{array}$ \\
\hline
\end{tabular}

$\left(^{*}\right)$ Apenas uma chapa e um tubo apresentaram trincas.

Com o objetivo de avaliar a robustez do projeto do aço, foram processadas no ciclo 4 , em ritmo normal de produção industrial, em torno de 1800 t de chapas, visando a atender ao grau X65 Sour Service nas espessuras de $29,65 \mathrm{~mm}$ e $32,45 \mathrm{~mm}$. A composição química média das chapas é apresentada na tabela 5 e os resultados de propriedades mecânicas e de resistência ao HIC são mostrados na figura 9.

Tabela 5. Composição química média do aço API 5L-X65 Sour Service-ciclo 4.

\begin{tabular}{c|c|c|c|c|c|c|c}
\hline $\mathbf{C}(\%)$ & $\mathbf{M n}(\%)$ & $\mathbf{P}(\mathbf{p p m})$ & $\mathbf{S}(\mathbf{p p m})$ & $\begin{array}{c}\mathbf{N b + V + T i} \\
(\%)\end{array}$ & $\begin{array}{c}\mathbf{C u + N i + M o} \\
(\%)\end{array}$ & $\mathbf{C e q}^{(1)}$ & $\mathbf{P c m}^{(2)}$ \\
\hline $\bar{X}=0,0342$ & $\bar{X}=1,20$ & $\bar{X}=0,009$ & $\bar{X}=0,0009$ & $\bar{X}=0,072$ & $\bar{X}=0,47$ & $\bar{X}=0,288$ & $\bar{X}=0,13$ \\
\hline$\sigma=0,0045$ & $\sigma=0,04$ & $\sigma=0,001$ & $\sigma=0,0002$ & $\sigma=0,004$ & $\sigma=0,02$ & $\sigma=0,008$ & $\sigma=0,005$ \\
\hline
\end{tabular}

(1) $\mathrm{Ceq}=\% \mathrm{C}+\% \mathrm{Mn} / 6+(\% \mathrm{Cr}+\% \mathrm{Mo}+\% \mathrm{~V}) / 5+(\% \mathrm{Ni}+\% \mathrm{Cu}) / 15$

(2) $P c m=\% C+\% S i / 30+(\% M n+\% C u+\% C r) / 20+\% N i / 60+\% M o / 15+\% V / 10+5 . \% B$

A análise da tabela 5 e da figura 9 permite verificar um baixo espalhamento na composição química e em todas as propriedades avaliadas, além de excelentes resultados de tenacidade no ensaio Charpy e de resistência ao HIC, confirmando a boa prática operacional utilizada e a robustez do projeto. Com relação à composição química destaca-se a garantia de obtenção de baixos teores de carbono, fósforo e enxofre, fundamental para o sucesso na fabricação desse tipo de aço. Os valores de limite de escoamento e de resistência das chapas se mantiveram próximos aos limites inferiores especificados para o tubo. Contudo, como discutido anteriormente, esses resultados não comprometem a aplicação, tendo em vista os ganhos nessas propriedades após a conformação em tubos. No caso do ensaio DWT, desconsiderando a fratura inversa, todas as chapas foram aprovadas, na temperatura de $-20^{\circ} \mathrm{C}$, com $100 \%$ de área dúctil. Cerca de $15 \%$ dos corpos de prova de DWTT ensaiados no ciclo 4 apresentaram fratura inversa. Isso, entretanto, não

\footnotetext{
* Contribuição técnica ao 69 Congresso Anual da ABM - Internacional e ao 14ํㅡㄹ ENEMET - Encontro Nacional de Estudantes de Engenharia Metalúrgica, de Materiais e de Minas, 21 a 25 de julho de 2014, São Paulo, SP, Brasil.
} 


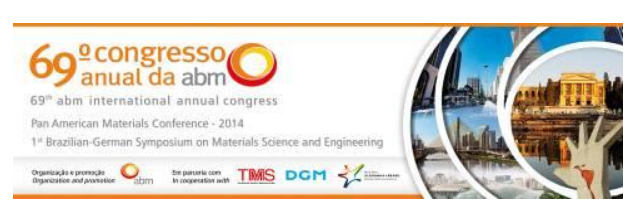

significa que as chapas que apresentaram o problema não tenham resistência à propagação da fratura frágil. Na opinião dos autores do presente trabalho, ao considerar a área de fratura inversa no cálculo da porcentagem de área dúctil, correse o risco de ser adotado um nível muito elevado de conservadorismo, o que pode implicar em reprovação de materiais perfeitamente adequados para a aplicação. Trabalhos posteriores serão apresentados com os resultados de DWTT desta família de aço, sendo gerados em dispositivo instrumentado, visando a demonstrar o elevado nível de energia necessário para a iniciação e propagação da fratura.
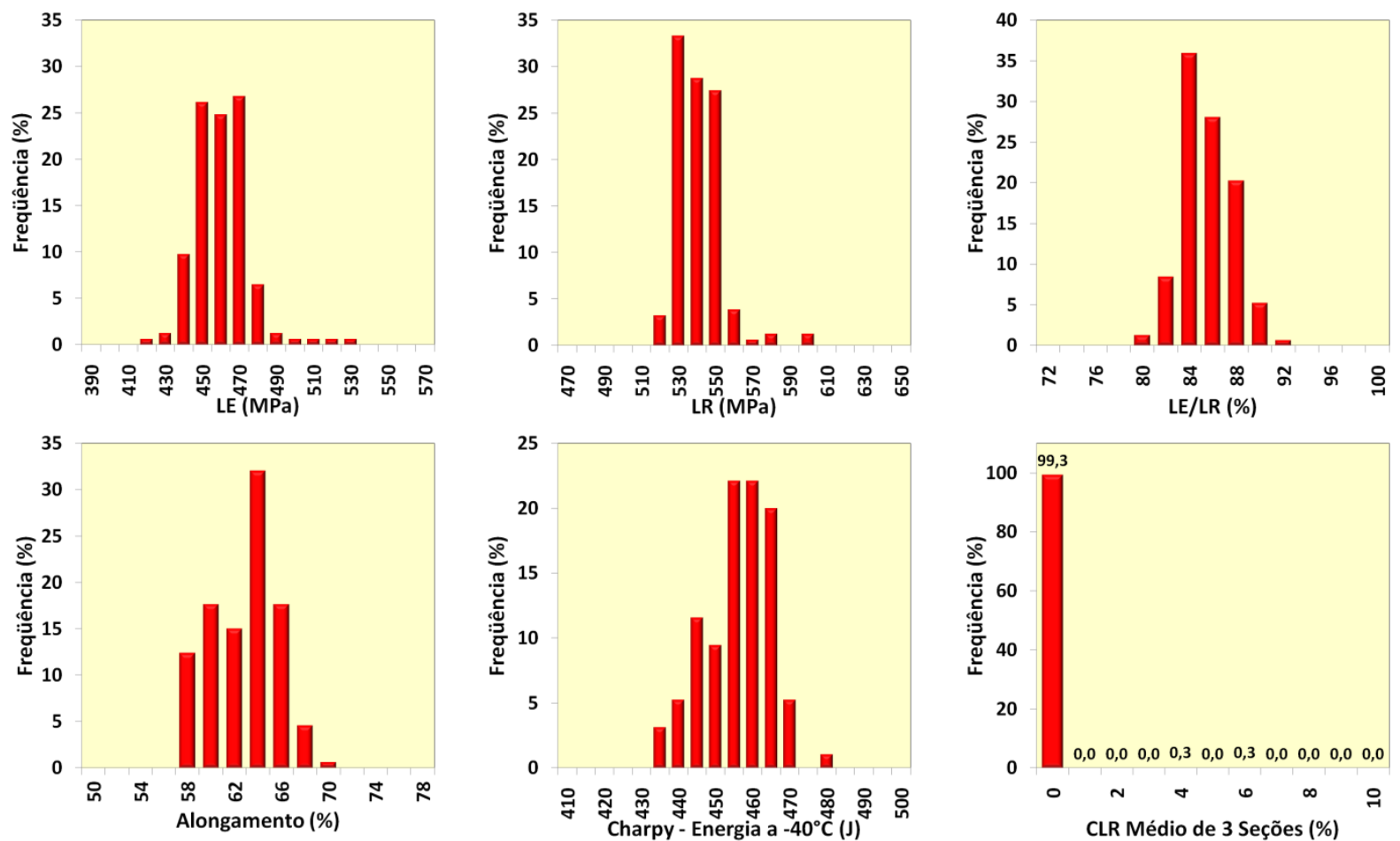

Figura 9. Resultados de propriedades mecânicas (ensaios realizados na direção transversal à de laminação) e de resistência ao HIC das chapas produzidas no ciclo 4

\section{CONCLUSÃO}

Os resultados obtidos indicam que o aço API 5L-X65 Sour Service desenvolvido atendeu ao mesmo tempo aos severos requisitos de resistência ao HIC e de propriedades mecânicas, em chapas e tubos de elevada espessura. Para o sucesso desse desenvolvimento foi necessário, além da definição adequada do projeto de liga do aço, o controle acurado das condições de processamento, desde a fabricação do aço líquido até a transformação das chapas em tubos. Em função desses resultados 0 aço foi homologado pela Petrobras para ser usado em gasodutos especificados para os projetos Rota 2 e Rota 3 do Pré-sal.

A produção em maior escala confirmou a boa prática operacional adotada e possibilitou concluir que o projeto do aço desenvolvido é suficientemente robusto para garantir a obtenção de faixas estreitas de propriedades.

\section{REFERÊNCIAS}

1 Hara T, Asahi H, Ogawa $\mathrm{H}$. Conditions of hydrogen-induced corrosion occurrence of X65 grade line pipe steels in sour environments. Corrosion. 2004;60(12):1113-21.

\footnotetext{
* Contribuição técnica ao 69 Congresso Anual da ABM - Internacional e ao 14ํㅡㄹ ENEMET - Encontro Nacional de Estudantes de Engenharia Metalúrgica, de Materiais e de Minas, 21 a 25 de julho de 2014, São Paulo, SP, Brasil.
} 


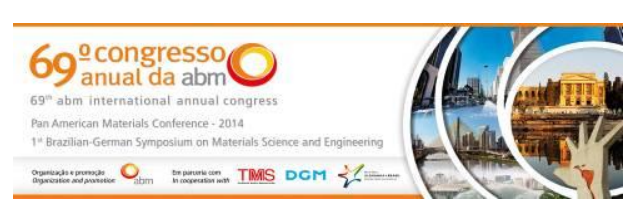

2 Stalheim DG, Hoh B. Guidelines for production of api pipelines steels suitable for hydrogen induced cracking (HIC) service applications. In: Proceedings of $8^{\text {th }}$ International Pipeline Conference; sep. 27-oct.1, 2010; Calgary, Canada. 2010. p.517-527.

3 Stallybrass C, Haase T, Konrad J, Bosch C, Kulgemeyer A. Alloy design for UOE linepipe material for standard and non-standard HIC conditions. In: Proceedings of the International Seminar Microalloyed Steels for Sour Service. Aug. 20-22, 2012; São Paulo, Brazil.

4 Moon J, Park C, Kim SJ - Influence of Ti addition on the hydrogen induced cracking of API 5 I X70 hot-rolled pipeline steel in acid sour media. Metals and Materials International. 2012;18(4):613-617.

5 Ishikawa N, Suzuki M, Matsushima T, Tsuji A, Kakihara S, Shikanai N, Awajiya H. Steel plate for line pipes and steel pipes. European Patent Office. EP 2224028 A1. 2009, 20p.

6 Bandyopadhyay PS, Kundu S, Ghosh S.K, Chatterjee S. Structure and properties of a low-carbon, microalloyed, ultra-high-strength steel. Metallurgical and Materials Transactions A. 2011;42A(4):1051-61.

7 Grimpe F, Meimeth S, Meuser H, Muthmann E, Liessem A, Stallybrass C. The development of high strength heavy plate for the pipe industry using modern experimental and numerical methods. In: Proceedings of International Conference on New Developments on Metallurgy and Applications of High Strength Steels; May 26-28, 2008; Buenos Aires, Argentina.

8 Hulka K. Sour gas resistant pipe steel. Niobium Information 18 [acesso em 20 fev. 2014]. Disponível em: http://www.cbmm.com.br/portug/sources/techlib/info/others_in_pdf/pdfs/Sour_Gas.pdf.

9 Hulka K, Gray JM. High Temperature Processing of Line-Pipe Steels. In: Proceedings of the International Symposium Niobium; 2001; Orlando, USA. 2001.

10 Koh SU, Jung HG, Kang KB, Park GT, Kim KY. Effect of microstructure on hydrogeninduced cracking of linepipe steels. Corrosion. 2008;64(7):574-585.

11 Park G, Koh SU, Jung HG, Kim KY. Effect of microstructure on the hydrogen trapping efficiency and hydrogen induced cracking of linepipe steel. Corrosion Science. 2008;50:1865-71.

12 Wang W, Shan Y, Yang K. Study of high strength pipeline steels with different microstructures. Materials Science and Engineering A. 2009;502:38-44.

13 Kim YM, Lee H, Yoo JY, Kim NJ. Microstructural characteristics of acicular ferrite in linepipe steels. In: Proceedings of The $15^{\text {th }}$ International Offshore and Polar Engineering Conference; Jun. 19-24, 2005; Seoul, Korea.

14 American Petroleum Institute. Specification for line pipe. 45a ed. ANSI/API Spec 5L; 2012.

15 DNV. Offshore standard for submarine pipeline systems. DNV-OS-F101; 2010.

16 British Standard. BS -7448 AMD 10543: Fracture mechanics toughness tests - Part 1: Method for determination of $\mathrm{K}_{\mathrm{Ic}}$ critical CTOD and critical $\mathrm{J}$ values of metallic materials. 1991 with corr. 2002.

17 NACE. Laboratory testing of metals for resistance to specific forms of environmental cracking in $\mathrm{H}_{2} \mathrm{~S}$ environments. NACE TM0177; 2005.

18 Hwang B, Lee S, Kim YM, Kim NJ, Yoo JY, Woo, CS. Analysis of abnormal fracture occurring during drop-weight tear test of high-toughness line-pipe steel. Materials Science and Engineering A. 2004;368:18-27.

19 Muraoka R, Ishikawa N, Endo S. Evaluation for abnormal fracture appearance in drop weight tear test with high toughness linepipe. In: Proceedings of $21^{\text {st }}$ International Conference on Offshore Mechanics and Artic Engineering; Jun. 23-28 2002; Oslo, Norway. 2002. p.129-136.

20 API. Recommended practice for conducting drop-weight tear tests on line pipe 5L3. $3^{\text {rd }}$ ed. API; 1996.

\footnotetext{
* Contribuição técnica ao 69 Congresso Anual da ABM - Internacional e ao 14ํㅡㄹ ENEMET - Encontro Nacional de Estudantes de Engenharia Metalúrgica, de Materiais e de Minas, 21 a 25 de julho de 2014, São Paulo, SP, Brasil.
} 